\title{
Complex Shear Modulus Estimation using Integration of LMS/AHI Algorithm
}

\author{
Quang-Hai Luong, Manh-Cuong Nguyen \\ Le Quy Don Technical University \\ Hanoi, Vietnam
}

\author{
TonThat-Long \\ Duc-Tan Tran \\ School of Electrical Engineering University of Engineering and Technology \\ International University \\ VNU-HoChiMinh City
}

HoChiMinh City, Vietnam

\begin{abstract}
Elasticity and viscosity of tissues are two important parameters that can be used to investigate the structure of tissues, especially detecting tumors. By using a force excitation, the shear wave speed is acquired to extract its amplitude and phase. This information is then used directly or indirectly to compute the Complex Shear Modulus (CSM consists of elasticity and viscosity). Among these methods, Algebraic Helmholtz Inversion (AHI) algorithm can be combined with the Finite Difference Time Domain (FDTD) model to estimate CSM effectively. However, this algorithm is strongly affected by measured noise while acquiring the particle velocity. Thus, we proposed a LMS/AHI algorithm which can estimate correctly CSM. A simulation scenario is built to confirm the performance of the proposed LMS/AHI algorithm with average error of $3.14 \%$.
\end{abstract}

Keywords-Shear wave; elasticity; viscosity; CSM estimation; least mean square; Algebraic Helmholtz Inversion

\section{INTRODUCTION}

Elasticity and viscosity of tissues are two important factors that can be exploited to detect tumors [1]. Many research work focused on elasticity [2]-[7] where ultrasonic Shear Wave Elasticity Imaging (SWEI) offers significant advantages over the other techniques in terms of reproducibility, quantification, elasticity contrast, and automatic shear wave generation. However, for deeply understanding about the tissue, various work have been developed to estimate both the elasticity and viscosity, which are briefly surveyed next. Breast needle biopsy is well-known in ultrasonic. In order to generate the shear wave, previous work applied force whose frequencies are low as $0.1 \mathrm{~Hz}$ and high as $10 \mathrm{kHz}$. Recently, they have used the excitation in the range of $50-250 \mathrm{~Hz}$ for simplification of the measurement. In this paper, only single frequency of $150 \mathrm{~Hz}$ is needed for the excitation. By applying the force at different spatial locations, the structure of the tissue can be investigated.

The relationship between the speed and absorption of shear wave to the corresponding CSM can be modeled by simple equations in [8]. If transient forces [9] are considered, the reflections are minimized. However, the affection of noise is worse than using harmonic forces [10]. If the harmonic needle vibration is used, compared with other force excitation techniques, larger amplitudes of shear wave can be obtained. Thus, a harmonic needle vibration is considered for excitation in this work. In 2004, Chen et al. exploited the relationship between the propagation speed and the vibrating frequency to build the shear wave speed dispersion, and then estimate the CSM [11]. In 2007, Zheng et al. used a linear Kalman filter for
CSM estimation over a frequency bandwidth [10]. The noise is reduced by this filter. Recently, some extended methods have been introduced in [10]-[13] where the authors needed to use multiple datasets of different vibration frequencies. In this paper, only a single-frequency excitation is needed, but still, the acquisition time is improved.

In order to detect tumor (if any) in the tissue, Tran et al. [14] used the maximum likelihood ensemble filter for $1 D$ heterogeneous tissue. However, the propagation model using wave equation in [12], [14], [15] is very simple, and it can not represent the actual propagation in heterogeneous tissue. In 2015, Qiuang et al. [16] proposed a method which uses Finite Element Method (FEM) to model the shear wave propagation in transversely isotropic, viscoelastic and incompressible media. However, the complexity of FEM is high. FDTD is more effective method with lower complexity than FEM. In [17], Orescanin et al. exploited FDTD model then used AHI algorithm to estimate CSM. However, there is a lack of deep investigation of noise in this work because AHI is strongly affected by noise.

In this paper, we introduce an integration of $\mathrm{AHI}$ and Least Mean Square (LMS) algorithms to estimate CSM. A shear wave generator at a single frequency of $100 \mathrm{~Hz}$ is excited at the origin $(0,0)$ by the vibrating needle. A linear array transducer is used to measure the particle velocity of shear wave at 120 spatial locations. At each point, the CSM from the noisy particle velocity of shear wave is then estimated by applying a specific LMS/AHI. Using the LMS/AHI can drastically reduce the complexity as compared to previous techniques. Finally, a scenario with a tumor and noise environment is studied to evaluate quality of the estimated CSM.

\section{METHOD}

\section{A. Shear Wave Propagation}

Generation of shear waves and measurements of the particle velocity are shown in Fig. 1. In this system, a needle is vibrated at a single frequency along the Z-axis, the share wave is then propagated in X-Y plane. After that, the particle velocity is acquired by using a Doppler ultrasound device [12].

In some previous work ( [12], [14], [15]), the wave (1) is used to compute the particle velocity $v(r, t)$ at a spatial 


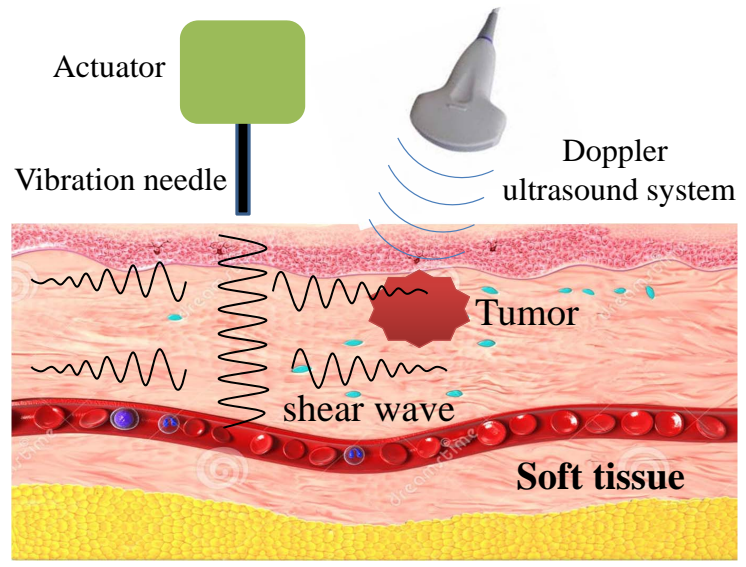

Fig. 1. Generation and measurement of shear wave.

location $r$ and time $t$.

$$
v(r, t)=\frac{1}{\sqrt{r-r_{0}}} A e^{-\alpha\left(r-r_{0}\right)} \cos \left[\omega t-k_{s}\left(r-r_{0}\right)-\phi\right],
$$

where $A$ is the vibration's amplitude of the needle, $r_{0}$ is the needle's spatial location, $\phi$ is the initial phase, $\alpha$ and $k_{s}$ are attenuation coefficient and wave number at spatial location $r$ respectively.

The formula (1) has the advantage of simplification. However, it cannot reflect the propagation of the shear wave in the real tissue, especially in a heterogeneous one. Thus, FDTD method is used, together with the assumption of cylindrical shear wave propagation along the radial axis and ignoring absorption of medium. Consequently, the particle velocity vector $v_{z}$ on a direction of the wave propagation $x$ in Cartesian coordinate relates to the stress tensor $\sigma_{z x}$, which can be described by the following (2) and (3) (from [17]):

$$
\begin{gathered}
\rho \partial_{t} v_{z}=\partial_{x} \sigma_{z x}, \\
\partial_{t} \sigma_{z x}=\left(\mu+\eta \partial_{t}\right) \partial_{x} v_{z},
\end{gathered}
$$

where $\partial_{t}$ represents a partial derivative operator $\partial / \partial_{t}$ applied to values to the right of the symbol, $\partial_{x}$ represents a partial derivative operator $\partial / \partial_{x}$ applied to values to the right of the symbol, $\rho$ is density of the tissue, $\mu$ and $\eta$ are the elasticity and viscosity of the tissue respectively.

Kelvin-Voigt model is applied to represent the CSM $G(x, \omega)$, which depends on the angle frequency of the vibration $\omega$ as follows

$$
G(x, \omega)=\mu(x)-i \omega \eta(x) .
$$

where $\mu$ is the elasticity and $\eta$ is the viscosity that need to be estimated. To discretize (2) and (3), the following notations will be used:

$$
\begin{gathered}
v_{z}(x, t)=v_{z}(i \Delta x, n \Delta t)=\left.v_{z}^{n}\right|_{i}, \\
\sigma_{z x}(x, t)=\sigma_{z x}(i \Delta x, n \Delta t)=\left.\sigma_{z x}^{n}\right|_{i},
\end{gathered}
$$

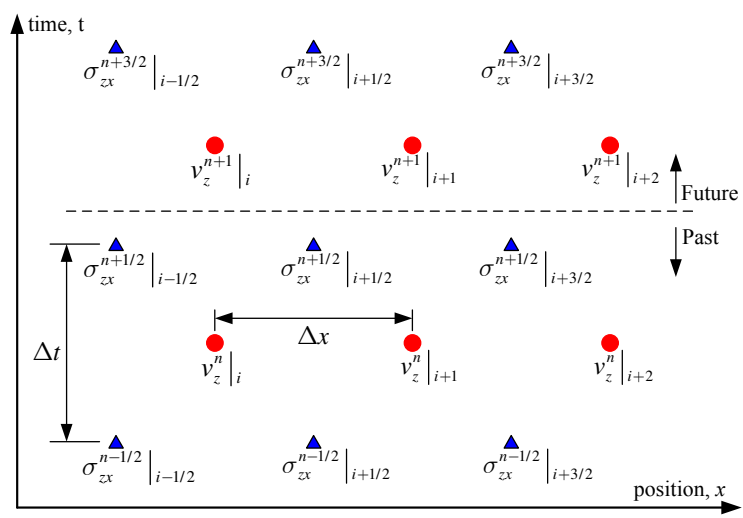

Fig. 2. Illustration of the stress tensor $\sigma$ and the particle velocity vector $v_{z}$ nodes in time and space.

where $\Delta x$ is the distance between continuous spatial locations, $\Delta t$ is the sampling period, the index $i$ is the spatial step, and the index $n$ is the temporal step, as shown in Fig. 2.

By using FDTD method, (2) and (3) are described as follows:

$$
\begin{aligned}
\left.v_{z}^{n+1}\right|_{i}=\left.v_{z}^{n}\right|_{i} & +\frac{\Delta t}{\rho \Delta x}\left(\left.\sigma_{z x}^{n+\frac{1}{2}}\right|_{i+\frac{1}{2}}-\left.\sigma_{z x}^{n+\frac{1}{2}}\right|_{i-\frac{1}{2}}\right), \\
\left.\sigma_{z x}^{n+\frac{1}{2}}\right|_{i+\frac{1}{2}}= & \left.\sigma_{z x}^{n-\frac{1}{2}}\right|_{i+\frac{1}{2}}+\frac{\mu \Delta t}{\Delta x}\left(\left.v_{z}^{n+1}\right|_{i+1}-\left.v_{z}^{n+1}\right|_{i}\right) \\
& +\frac{\eta}{\Delta x}\left(\left.v_{z}^{n+1}\right|_{i+1}-\left.v_{z}^{n+1}\right|_{i}\right) \\
& -\frac{\eta}{\Delta x}\left(\left.v_{z}^{n}\right|_{i+1}-\left.v_{z}^{n}\right|_{i}\right),
\end{aligned}
$$

\section{B. Signal Enhancement using Least Mean Square Algorithm}

Adaptive filters attracts a great attention due to its property of self adjusting their coefficients [18]. For the signal enhancement, the output signal is obtained from a noisy input signal and an adaptation process. The filter coefficients are adjusted in order to minimize a desired cost function. There are a lot of filter structures and adaptive algorithms that have been developed in recent decades [19]. In this paper, we design a transversal adaptive filter to reduce the noise from the noisy particle velocity which is acquired from the Doppler ultrasound system, as shown in Fig. 3.

In Fig. 3, a particle velocity signal, represented as $d(n)$, is transmitted into the tissue which is affected by noises, represented as $v(n)$. Together, they form a noisy signal $v_{z}(n)$ which is described by

$$
v_{z}(n)=d(n)+v(n) .
$$

This noisy signal $v_{z}(n)$ is applied as an input to the adaptive filter to extract the estimate of the desired signal with minimum error using various adaptive methods such as LMS, Normalised Least Mean Square (NLMS), Root Mean Square (RMS) algorithms, etc. When the estimate of noise equals or approximates the $v[n](y(n)=v(n))$, the error 


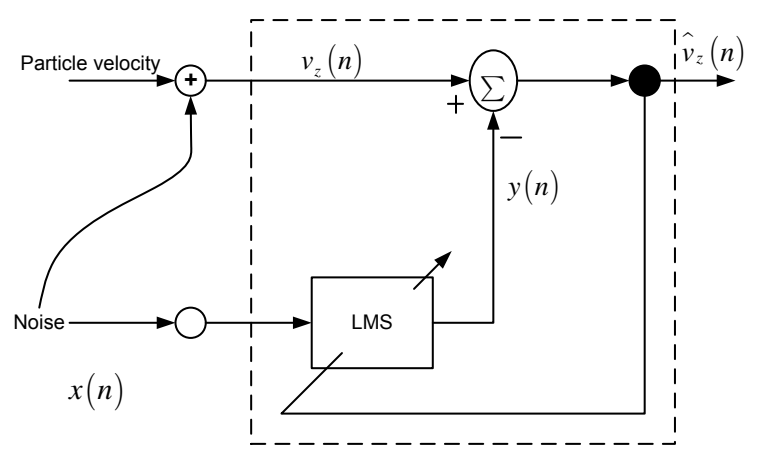

Fig. 3. Using LMS filter to enhance the particle velocity.

signal is approximately the filtered speech signal $d(n)$ because $e(n)=d(n)+v(n)-v(n)=d(n)$. The output of the adaptive filter or filtered signal $\widehat{v}_{z}(n)=e(n)$.

The input signal is sampled and it forms a vector containing $N$ samples.

$$
v_{z}(n)=\left[\begin{array}{llll}
v_{z}(0) & v_{z}(1) & . . & v_{z}(N-1)
\end{array}\right],
$$

The corresponding estimated value of the desired signal is $\widehat{v}_{z}(n)$. The coefficients of the filter are represented as

$$
w(n)=\left[\begin{array}{llll}
w(0) & w(1) & . . & w(L)
\end{array}\right] .
$$

where $L$ represents the order of the filter. The filter coefficients, alternatively called as weights $w(n)$, are adjusted every time in such a way that the Mean Square Error (MSE) is minimized. There are many well-known adaptive algorithms that can accomplish this weight adjustment. Among them, the LMS algorithm has a simple filter weight update mechanism, which has a fast rate of convergence if an optimal step size is used.

The LMS algorithm can be summarized in Algorithm 1 below:

\section{Algorithm 1: LMS Algorithm for Shear Wave Acqui- sition}

Step 1: Initialize the step size $\mu$, filter order $L$, and noise variance.

Step 2: Initialize the filter coefficients $w(n)=0$.

Step 3: For $n=0,1,2$, .

3.1. Compute the filter output

$y(n)=w(n) * x(n)$

3.2. Compute the error in estimation

$e(n)=v_{z}(n)-y(n)$

3.3. Compute the updated tap-weight

$w(n+1)=w(n)+\mu e(n) x(n)$

3.4. Compute the denoised signal by assigning

$\widehat{v}_{z}(n)=e(n)$

3.5. Iterate till end of the signal

end

C. Direct Inversion using Least Mean Square/Algebraic Helmholtz Inversion Algorithm to Estimate the CSM

After reducing the noise from acquired particle velocity, the AHI algorithm [20] is used to compute the CSM. For a small volume, it is assumed that the viscoelastic property of the tissue is isotropic. Thus, there is negligible compression applied to the tissue by the needle, as a result, the particle velocity vector $v_{z}$ can be described by the Navier wave equation in a homogeneous solid. We combine (2) and (3) to obtain

$$
\rho \frac{\partial^{2} v_{z}}{\partial t^{2}}=G^{\prime}(x, t) \nabla^{2} v_{z},
$$

where $G^{\prime}(x, t)$ is the CSM in time domain and $\nabla^{2} v_{z}$ is Laplace operator of $v_{z}$ which is defined as $\nabla^{2} v_{z}=\partial^{2} v_{z} / \partial x^{2}$.

AHI algorithm is applied to solve (12), which then becomes the Helmholtz equation

$$
\left.\left(\frac{G(x, \omega)}{\rho} \nabla^{2}+\omega^{2}\right) V_{z}(x, \omega)\right|_{\omega=\omega_{0}}=0,
$$

where $G(x, \omega)$ is the CSM in frequency domain and defined in (4), $V_{z}(x, \omega)$ is the temporal Fourier transform of the particle velocity $v_{z}(x, t), V_{z}(x, \omega)=F_{t}\left\{v_{z}(x, t)\right\}$, and $\omega_{0}$ is the angular frequency $\omega_{0}=2 \pi f_{0}$. From (13), it can be seen that the CSM can be estimated directly as

$$
\begin{aligned}
& \mu(x)=\Re\left\{\frac{-\rho \omega_{0}^{2} V_{z}\left(x, \omega_{0}\right)}{\nabla^{2} V_{z}\left(x, \omega_{0}\right)}\right\}, \\
& \eta(x)=\Im\left\{\frac{-\rho \omega_{0} V_{z}\left(x, \omega_{0}\right)}{\nabla^{2} V_{z}\left(x, \omega_{0}\right)}\right\},
\end{aligned}
$$

where $V_{z}\left(x, \omega_{0}\right)$ is computed by using Fourier transform at the specific angular frequency $\omega_{0} ; \nabla^{2} V_{z}\left(x, \omega_{0}\right)$ is computed by using the function Discrete Laplacian (The MathWorks) $\operatorname{del} 2\left(V_{z}\left(x, \omega_{0}\right)\right)$ which returns a discrete approximation of Laplaces differential operator applied to $V_{z}\left(x, \omega_{0}\right)$.

The proposed LMS/AHI for CSM estimation is summarized in Algorithm 2.

\section{Algorithm 2: LMS/AHI Algorithm for CSM Estima- tion}

Step 1: Set up the simulation scenario.

Step 2: Select the excitation frequency $f_{0}=150 \mathrm{~Hz}$.

Step 3: Generate shear waves by vibrating the needle.

Step 4: Acquire the noisy particle velocity at 120 spatial locations.

Step 5: Estimate the noise variance from the noisy signal corrupted by the additive white noise.

Step 6: Reduce the noise from noisy particle velocity using LMS filter as shown in Algorithm 1.

Step 7: Discard the transient parts of the filtered signal and keep the steady-state of the filtered particle velocity.

Step 8: Compute the temporal Fourier transform of the filtered signal.

Step 9: Estimate each CSM in spatial locations using (14).

Step 10: Evaluate the estimation performance.

end

\section{RESUlTS AND DisCUSSIONS}

In order to verify the proposed method LMS/AHI, a simulation scenario is built where $1 \mathrm{D}$ heterogeneous tissue is $12 \mathrm{~mm}$ in length and the distance between two continuous spatial locations is $0.1 \mathrm{~mm}$. At each spatial location, we consider 20000 samples. The vibrating frequency is chosen as $150 \mathrm{~Hz}$; the amplitude of the vibration is $2 \mathrm{~mm}$. The elasticity and viscosity of the tissue are shown in Table I. 
TABLE I. SimULATION SCENARIO

\begin{tabular}{|c||c||c|}
\hline Spatial locations & Elasticity [Pa] & Viscosity [Pa.s] \\
\hline $1-29$ & 650 & 0.1 \\
\hline $30-50$ & 900 & 0.35 \\
\hline $51-120$ & 650 & 0.1 \\
\hline
\end{tabular}

It can be seen that there is an inclusion (from the $30^{\text {th }}$ to $50^{t h}$ spatial locations) in this tissue.

Fig. 4 and 5 present the particle velocities in term of time at the $15^{\text {th }}$ and $60^{\text {th }}$ points, respectively. They are sinusoidal functions of time in two cases: noisy and filtered signals. The filtered signal can be divided into two parts: transient part which is still affected by noise and the steady-state part where the noise was filtered out completely. It is obviously that the amplitude at the $15^{\text {th }}$ spatial location is larger than that of the $60^{t h}$ one. However, the power of the additive noise is the same for all $L=120$ spatial locations. It is noted that the noise would strongly affect the CSM estimation due to the limitation of AHI methodology.

Fig. 6 shows the particle velocity vs. spatial locations where the undeniable role of measured noise is also illustrated. Furthermore, as shown in this figure, the power of the additive noise is the same for every spatial location.

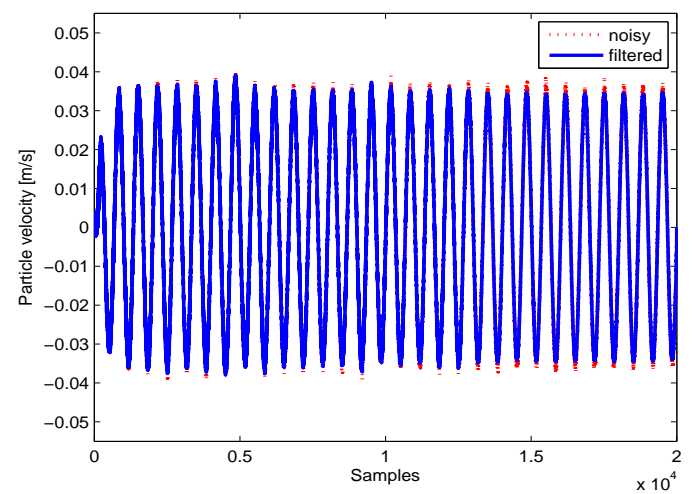

Fig. 4. The particle velocity in time at the $15^{\text {th }}$ point.

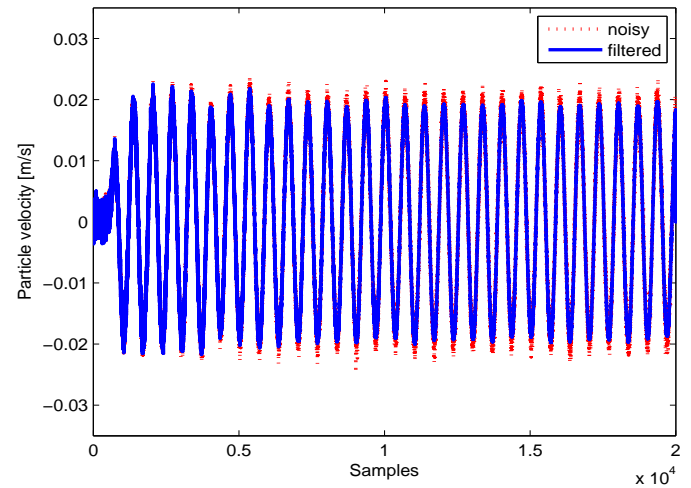

Fig. 5. The particle velocity in time at the $60^{\text {th }}$ point.

Without noise filtering, CSM can not be estimated. Thus, the following three cases, which are shown in Fig 7 and 8, are concerned: ideal estimation, LMS/AHI without removing

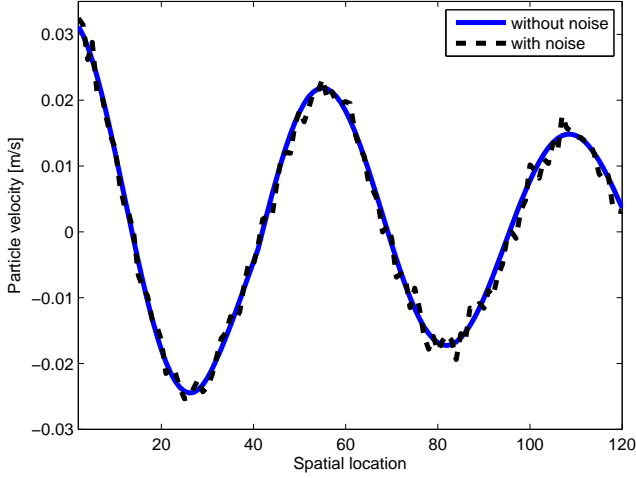

Fig. 6. The particle velocity vs. spatial locations.

the transient parts of the filtered signal, and the proposed LMS/AHI (i.e removing the transient part and keep the steadystate of the filter signal). Without removing the transient part, the estimation can not trace well the ideal ones. The reason is that the noise is still existed in this part and it affects both calculations of $v_{z}\left(x, \omega_{0}\right)$ and $\nabla^{2} v_{z}\left(x, \omega_{0}\right)$. By cutting the transient parts of the filtered signal and keep the steadystate of the filtered particle velocity, the calculations of both $v_{z}\left(x, \omega_{0}\right)$ and $\nabla^{2} v_{z}\left(x, \omega_{0}\right)$ are improved. Hence, the elasticity and viscosity can trace well the ideal ones.

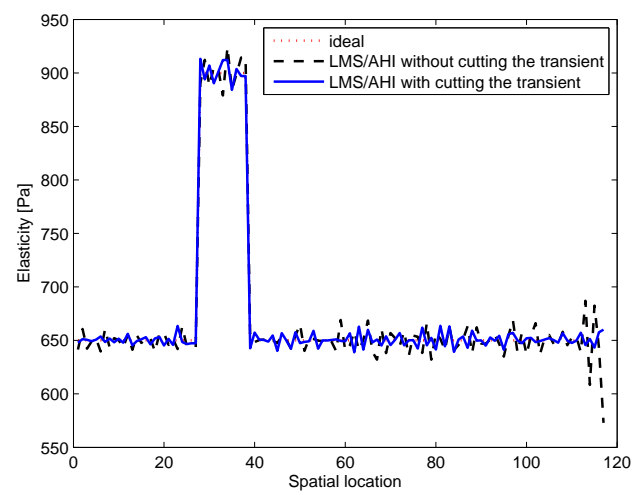

Fig. 7. The estimated elasticity.

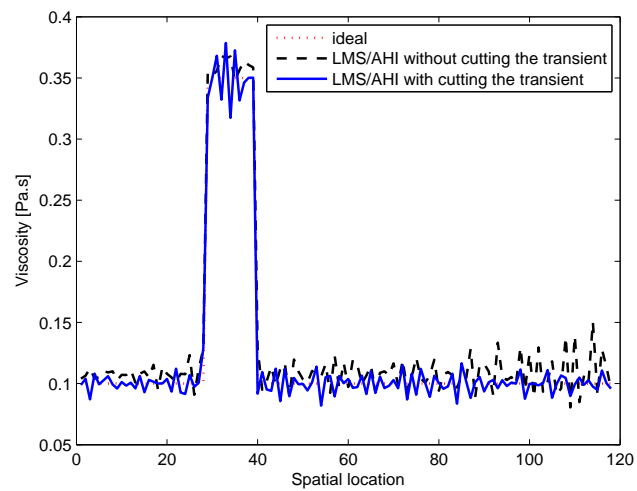

Fig. 8. The estimated viscosity. 
To quantify the efficiency of the proposed LMS/AHI algorithm, the error between the ideal CSM $(\mu, \eta)$ and the estimated $\operatorname{CSM}(\hat{\mu}, \hat{\eta})$ on different ranges of the tissue is computed. The normalized error can be defined as:

$$
\begin{aligned}
& \epsilon_{\mu}=\frac{1}{L} \sum_{i=1}^{L} \frac{\mid \underline{\mu_{i}-\hat{\mu}_{i} \mid}}{\mu_{i}} \\
& \epsilon_{\eta}=\frac{1}{L} \sum_{i=1}^{L} \frac{\left|\eta_{i}-\hat{\eta}_{i}\right|}{\eta_{i}}
\end{aligned}
$$

The results are shown in Table II. It can be seen that the quality of estimation is significantly improved by cutting the transient part of the filtered particle velocity. The transient part, which is still affected by noise, would affect the CSM estimation. The efficiency of the proposed LMS/AHI algorithm is confirmed by this error performance. As indicated in Table II, with LMS/AHI algorithm after cutting the transient part, we can achieve the average error of both elasticity and viscosity as $3.14 \%$.

\section{TABLE II. ERROR OF THE CSM ESTIMATION}

\begin{tabular}{|c||c||c|}
\hline Spatial locations & $\begin{array}{c}\text { Error } \\
\text { for elasticity [\%] }\end{array}$ & $\begin{array}{c}\text { Error } \\
\text { for viscosity [\%] }\end{array}$ \\
\hline LMS/AHI without cutting the transient & 1.27 & 10.39 \\
\hline LMS/AHI with cutting the transient & 0.64 & 5.64 \\
\hline
\end{tabular}

The results in this study are compared with some previous results. In [14], [15] and [12], authors used the MLEF (Maximum Likelihood Ensemble Filter) to estimate CSM. The error of estimation in [14], [15] is less than $10 \%$ for both elasticity and viscosity while in [12], the error of elasticity estimation is less than $2 \%$ and the error of viscosity estimation is within 5\%. However, in the studies [14], [15] and [12], the particle velocity of shear wave is modelled following the basic wave propagation equation which is only suitable for a homogeneous medium. In this paper, we have applied FDTD method to model the particle velocity of shear wave and this is correct with a heterogeneous medium (i.e. tissues). In addition, an adaptive filter (LMS) has been added for denoising the measured particle velocity of shear wave before estimating CSM using AHI algorithm. This is an advantage of this paper comparing with [17] which applied only AHI algorithm to estimate CSM.

\section{CONCLUSION}

A method for 1D estimation of CSM in tissues have been proposed successfully in this paper. The proposed method has used LMS/AHI to estimate CSM at each point in the medium with good estimation error of $3.14 \%$. In the experiment, only a single vibration frequency $(150 \mathrm{~Hz})$ is used. The propagation of shear wave is modeled using FDTD method with good accuracy and low complexity compared to FEM. The noise in the particle velocity is filtered using a specific LMS filter. CSM is then estimated directly using AHI after removing the transient part of filtered signal. In the future work, we will improve the accuracy of the CSM estimation, especially for the viscosity of tissues. The target will be able to distinguish between objects and the medium when the CSM of the objects are not much greater than that of the medium (i.e. early tumor detection).

\section{ACKNOWLEDGMENT}

This work was supported by the Asia Research Center (ARC), Vietnam National University, code CA.17.6A.

\section{REFERENCES}

[1] J. Bercoff, A. Criton, C. Bacrie, J. Souquet, M. Tanter, J. Gennisson, T. Deffieux, M. Fink, V. Juhan, A. Colavolpe et al., "ShearWave Elastography A new real time imaging mode for assessing quantitatively soft tissue viscoelasticity," in Ultrasonics Symposium, 2008. IEEE, 2008, pp. 321-324.

[2] A. P. Sarvazyan, O. V. Rudenko, S. D. Swanson, J. B. Fowlkes, and S. Y. Emelianov, "Shear wave elasticity imaging: a new ultrasonic technology of medical diagnostics," Ultrasound in medicine \& biology, vol. 24, no. 9, pp. 1419-1435, 1998.

[3] J.-L. Gennisson, T. Deffieux, M. Fink, and M. Tanter, "Ultrasound elastography: principles and techniques," Diagnostic and interventional imaging, vol. 94, no. 5, pp. 487-495, 2013.

[4] G. Ferraioli, P. Parekh, A. B. Levitov, and C. Filice, "Shear wave elastography for evaluation of liver fibrosis," Journal of Ultrasound in Medicine, vol. 33, no. 2, pp. 197-203, 2014.

[5] Y. Kobayashi, M. Tsukune, T. Miyashita, and M. G. Fujie, "Simple empirical model for identifying rheological properties of soft biological tissues," Physical Review, vol. 95, no. 2, 2017.

[6] S. Woo, S. Y. Kim, M. S. Lee, J. Y. Cho, and S. H. Kim, "Shear wave elastography assessment in the prostate: an intraobserver reproducibility study," Clinical imaging, vol. 39, no. 3, pp. 484-487, 2015.

[7] W. Zhang and S. Holm, "Estimation of shear modulus in media with power law characteristics," Ultrasonics, vol. 64, pp. 170-176, 2016.

[8] J. F. Greenleaf, M. Fatemi, and M. Insana, "Selected methods for imaging elastic properties of biological tissues," Annual review of biomedical engineering, vol. 5, no. 1, pp. 57-78, 2003.

[9] L. Sandrin, B. Fourquet, J.-M. Hasquenoph, S. Yon, C. Fournier, F. Mal, C. Christidis, M. Ziol, B. Poulet, F. Kazemi et al., "Transient elastography: a new noninvasive method for assessment of hepatic fibrosis," Ultrasound in Medicine and Biology, vol. 29, no. 12, pp. 1705-1713, 2003.

[10] Y. Zheng, S. Chen, W. Tan, R. Kinnick, and J. Greenleaf, "Detection of tissue harmonic motion induced by ultrasonic radiation force using pulse-echo ultrasound and kalman filter," IEEE Transactions on Ultrasonics, Ferroelectrics, and Frequency Control, vol. 54, no. 2, pp. 290-300, 2007.

[11] S. Chen, M. Fatemi, and J. F. Greenleaf, "Quantifying elasticity and viscosity from measurement of shear wave speed dispersion," The Journal of the Acoustical Society of America, vol. 115, no. 6, pp. 27812785, 2004.

[12] M. Orescanin and M. F. Insana, "Model-based complex shear modulus reconstruction: A Bayesian approach," in Ultrasonics Symposium. IEEE, 2010, pp. 61-64.

[13] Y. Wang and M. F. Insana, "Viscoelastic properties of rodent mammary tumors using ultrasonic shear-wave imaging," Ultrasonic imaging, vol. 35, no. 2, pp. 126-145, 2013.

[14] T. Tran-Duc, Y. Wang, N. Linh-Trung, M. N. Do, and M. F. Insana, "Complex Shear Modulus Estimation Using Maximum Likelihood Ensemble Filters," in 4th International Conference on Biomedical Engineering in Vietnam. Springer Berlin Heidelberg, 2013, pp. 313316.

[15] N. T. Hao, T. Thuy-Nga, V. Dinh-Long, T. Duc-Tan, and N. LinhTrung, "2D Shear Wave Imaging Using Maximum Likelihood Ensemble Filter," in International Conference on Green and Human Information Technology (ICGHIT), 2013, pp. 88-94.

[16] B. Qiang, J. Brigham, S. Aristizabal, J. Greenleaf, X. Zhang, and M. Urban, "Modeling transversely isotropic, viscoelastic, incompressible tissue-like materials with application in ultrasound shear wave elastography," Physics in medicine and biology, vol. 3, no. 60, pp. 12891306, 2015. 
[17] M. Orescanin, Y. Wang, and M. F. Insana, "3d fdtd simulation of shear waves for evaluation of complex modulus imaging," IEEE transactions on ultrasonics, ferroelectrics, and frequency control, vol. 58, no. 2, pp. 389-398, 2011.

[18] S. Haykin, "Adaptive filter theory," 2008.
[19] M. H. Hayes, Statistical digital signal processing and modeling. John Wiley \& Sons, 2009.

[20] S. Papazoglou, U. Hamhaber, J. Braun, and I. Sack, "Algebraic helmholtz inversion in planar magnetic resonance elastography," Physics in medicine and biology, vol. 53, no. 12, p. 3147, 2008. 